\title{
Microenvironment of adult T-cell leukemia/lymphoma-associated nodal lesions
}

\author{
Koichi Ohshima $\cdot$ Daisuke Niino $\cdot$ Kennosuke Karube
}

Received: 6 November 2013/Revised: 15 January 2014/ Accepted: 15 January 2014/Published online: 31 January 2014

(c) The Japanese Society of Hematology 2014

\begin{abstract}
Adult T-cell leukemia/lymphoma (ATLL) is a human malignancy associated with the retrovirus, human T-cell leukemia virus type I (HTLV-I). In a previous series of studies, our group and others characterized the histopathological changes in HTLV-I-associated lymph node lesions. In addition to the pleomorphic and anaplastic large cell types of typical ATL lymphoma, we identified lymph nodes with an unusual Hodgkin's disease-like histology (Hodgkin-like ATLL) in HTLV-I-positive patients, with Hodgkin-like ATLL showing prodromal clinical features. We also reported HTLV-I-associated lymphadenitis, characterized by nonneoplastic HTLV-I-associated lymph node lesions. It has become clear that the biological and clinical behavior of malignant lymphoma is not only determined by the properties of the lymphoma cells themselves, but also largely by the interaction of these cells with their nonmalignant microenvironment. In this review, we discuss the pathological variations of microenvironments, which are important for clarification of the histological features associated with HTLV-I.
\end{abstract}

Keywords ATLL $\cdot$ Pathology $\cdot$ Microenvironment

\section{Introduction}

In the early $1980 \mathrm{~s}$, the first human retrovirus, human T-cell leukemia virus type I (HTLV-I) was isolated and its characterization opened up the new field of human viral oncology. Adult T-cell leukemia/lymphoma (ATLL) is clinically characterized by the appearance of characteristic

K. Ohshima $(\varangle) \cdot$ D. Niino $\cdot$ K. Karube

Department of Pathology, School of Medicine, Kurume

University, Asahimati 67, Kurume 830-0011, Japan

e-mail: ohshima_kouichi@med.kurume-u.ac.jp flower cells, a rapid clinical course, occasional skin lesions, lymphadenopathy, and hepatosplenomegaly $[1,2]$. In addition, HTLV-I infection is associated with autoimmune and reactive disorders, such as HTLV-I-associated myelopathy (HAM) and uveitis, and is also related to immunodeficient infectious diseases. Pathological findings of ATLL in the lymph nodes, skin, liver, and other organs have been described elsewhere [3]. Histologically, ATLL usually exhibits the characteristics of peripheral T-cell lymphoma, with a diffuse proliferation of tumor cells varying in size and form combined with the appearance of cerebriform giant cells. In ATLL lymph nodes, the pathological features change from those of lymphadenitis to Hodgkin's-like features and of lymphoma. Non-neoplastic HTLV-1 associated lymph node lesions are thus considered to be associated with HTLV-1 associated lymphadenitis, while Hodgkin's-like histological features are associated with the initial stages of ATLL $[4,5]$.

It has also become clear that the biological and clinical behavior of malignant lymphoma is not only determined by the properties of the lymphoma cells themselves, but are also and largely by the interaction of the lymphoma cells with their nonmalignant microenvironment. The composition and functional status of the tumor microenvironment vary greatly among different classes of malignant lymphoma and may provide both growth supportive and growth suppressive signals via components of the adaptive and innate immune response. Recently, the tumor promoting features of innate and adaptive immune responses have been recognized. For instance, the indirect growth support by suppression of cytotoxic $\mathrm{T}$ cells by FoxP3 ${ }^{+}$ regulatory $\mathrm{T}$ cells and innate immune cells and the direct growth promoting features of innate immune cells as well as humoral immune responses are considered to be involved in epithelial tumors [6]. 
Macrophages that infiltrate tumor tissues are referred to as tumor-associated macrophages (TAMs) and are closely involved in tumorigenesis by inducing angiogenesis, immunosuppression, and invasion. Many studies of TAMs in human malignant tumors have been published since 2000, and they have demonstrated that TAMs are associated with histological grade and clinical prognosis in many kinds of tumors including hematological malignancies. As for malignant lymphomas, it has been reported that TAMs of T-cell-/histiocyte-rich large B-cell lymphoma (THRLBCL) are recruited within neoplastic infiltrates mainly by clonederived macrophage chemotactic proteins (MCPs) and represent a major component of the infiltrates [7].

\section{Morphology and characters of lymph node lesions}

Histopathological examination of the HTLV-I associated lymph nodes usually, although not uniformly, discloses a pleomorphic type. In addition to overt ATLL, some patients with pre-overt ATLL show a Hodgkin's diseaselike morphology and lymph nodes in non-neoplastic carriers with features of lymphadenitis [4, 5].

Pleomorphic (medium and large cell) type

The medium and large tumor cells vary in size and clearly show nuclear irregularity. Giant cells, with cerebriform, Reed-Sternberg type or bizarre nuclei, are frequently seen in these tissues (Fig. 1) and are nodal lesions typical of ATLL. Immunohistological evidence indicates that these tumor cells contain peripheral $\mathrm{T}$ cell phenotypes $\left(\mathrm{CD}^{-}\right.$, $2^{+}, 3^{+}, 45 \mathrm{RO}^{+}$and usually $\mathrm{CD}^{+}$) while receptor gene analysis has identified a rearrangement and/or deletion of the TCR gene $C \beta$ and/or J $\gamma$. Proviral HTLV-1 DNA bands have been detected in all cases [8].

Pleomorphic small cell type

Histologically, these tumor cells are as large as or slightly larger than normal blood lymphocytes (Fig. 1) and show mild nuclear irregularities with only a few cells displaying mitotic features. Tumor cells are of the peripheral $\mathrm{T}$ cell phenotype and with rearrangement and/or deletion of the TCR genes. Proviral HTLV-1 DNA bands have been detected in all cases [8].

Anaplastic large cell type

These tumor cells are much larger than the cells of large cell lymphoma and show a uniform pattern of cell proliferation. Tumor cells with prominent nucleoli and an abundant cytoplasm have been detected as have multinucleated giant cells such as Reed-Sternberg cells (Fig. 1). Tumor cells containing the CD30 antigen and peripheral T cell phenotype as well as rearrangement and/or deletion of the TCR genes have been detected, with proviral HTLV-1 DNA bands present in all cases [8].
Fig. 1 Histology of

pleomorphic (medium and large cell) type, pleomorphic small cell type, anaplastic large cell type and AILT-like ATLL. a The pleomorphic (mediumsized and large cell) type show a diffuse proliferation of atypical medium-sized to large lymphoid cells with irregular nuclei, intermingled with cerebriform giant cells (in the center). b The lymph nodes of pleomorphic small cell type show a diffuse proliferation of atypical medium-sized to small lymphoid cells. c Anaplastic large cell type show a diffuse proliferation of atypical large lymphoid cells with prominent nucleoli. d AILT-like ATLL shows proliferation of high endothelial venules with various infiltrating inflammatory cells

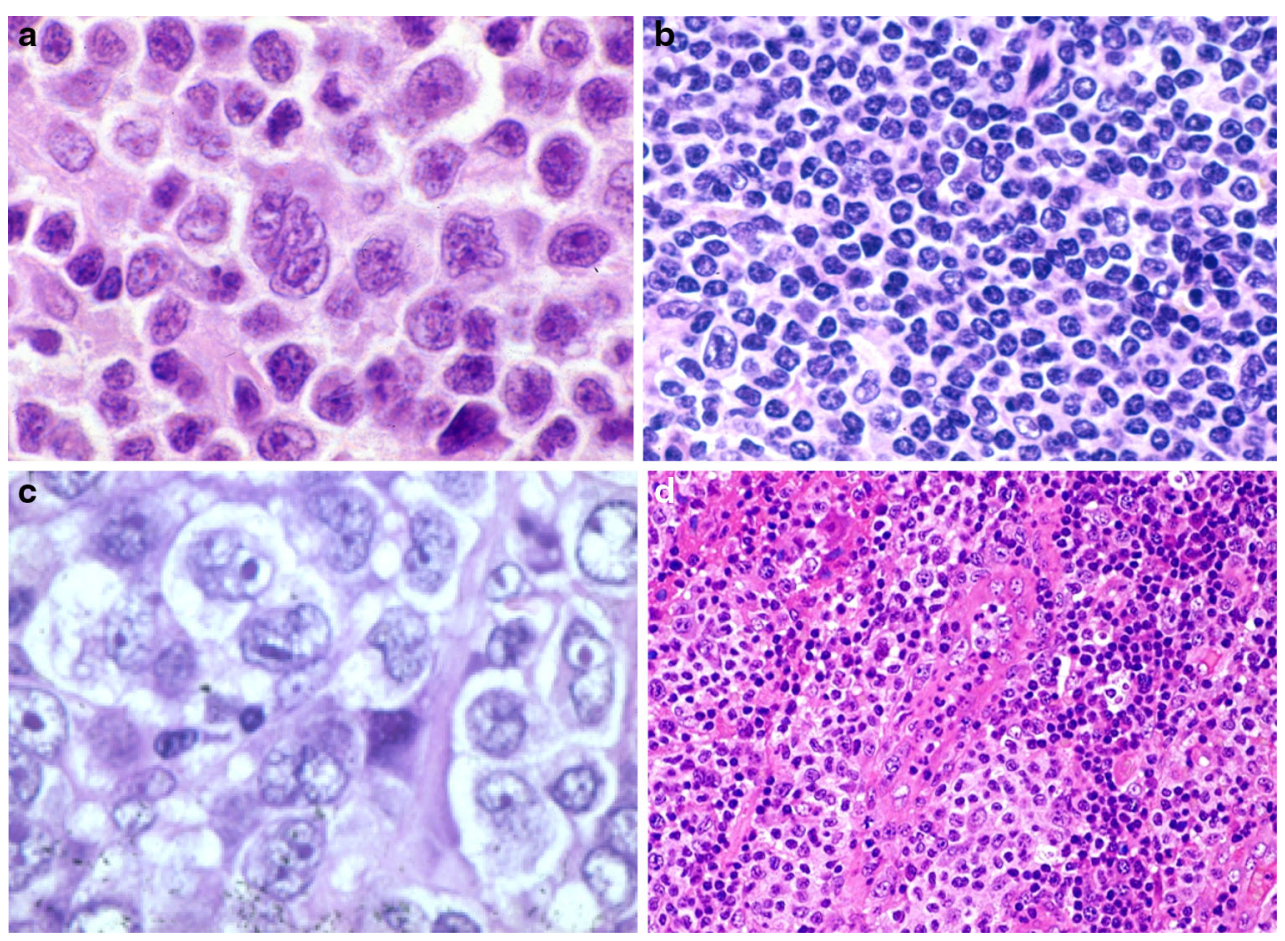




\section{AILT-like ATLL}

This rare morphological variant of ATLL includes the angioimmunoblastic T cell lymphoma (AILT)-like type. The lymph nodes examined to date have shown proliferation of high endothelial venules and the presence of various infiltrating inflammatory cells including plasma cells and eosinophils (Fig. 1). The lymphoma cells are medium to large in size with clear cytoplasm. Tumor cells are of the peripheral $\mathrm{T}$ cell phenotype with rearrangement and/or deletion of the TCR genes. Proviral HTLV-1 DNA bands have been detected in all cases with this rare type [9].

\section{Lymphadenitis type}

Histological examination of the lymph nodes of HTLV-I associated lymphadenitis reveals a preserved nodal architecture with small lymph follicles, enlargement of the paracortex, and diffuse infiltration of small or intermediate-size lymphocytes (Fig. 2), with the latter cells showing slight nuclear irregularities. Immunohistochemically stained sections have shown proliferating small to intermediate-size lymphocytes which possess a peripheral helper/inducer $\mathrm{T}$ cell phenotype $\left(\mathrm{CD} 1^{-}, 2^{+}, 3^{+}, 4^{+}, 8^{-}\right)$, while no cases have showed rearrangement or deletion of the $\mathrm{T}$ cell receptor gene $\mathrm{C} \beta$ and $\mathbf{J} \gamma$, or rearrangement of the immunoglobulin heavy chain gene $(\mathrm{JH})$ has not been detected in any cases. Except for a few cases with oligoclonal bands, no monoclonal proviral DNA bands have been detected. However, these bands were weaker than those of typical ATLL, probably because of the small population of HTLV-I integrated lymphocytes [5].

\section{Hodgkin's-like type}

These lymph nodes exhibit a relatively preserved nodal architecture with diffuse infiltration of small or mediumsized lymphocytes with mild nuclear irregularities. Small foci or clusters of a few aggregated giant cells with irregularly lobulated, highly convoluted, Reed-Sternberg or Hodgkin's cell-like nuclei are scattered throughout the expanded paracortex (Fig. 2). The giant cells occasionally display mitotic features. Immunohistological analysis has demonstrated that the proliferating small to medium-sized

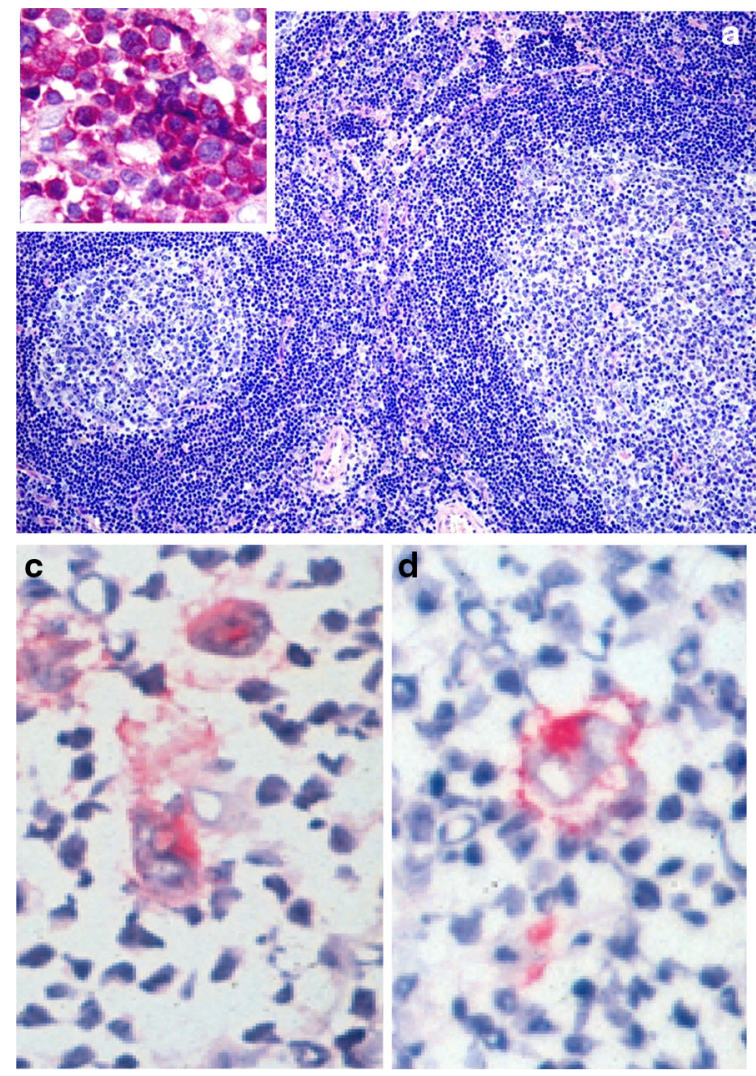

Fig. 2 Histology of HTLV-I associated lymphadenitis and Hodgkinlike ATLL. a The lymph nodes of HTLV-I associated lymphadenitis show enlarged paracortex with a diffuse infiltration of lymphocytes, which express the CD4 antigen (window). b The lymph nodes of

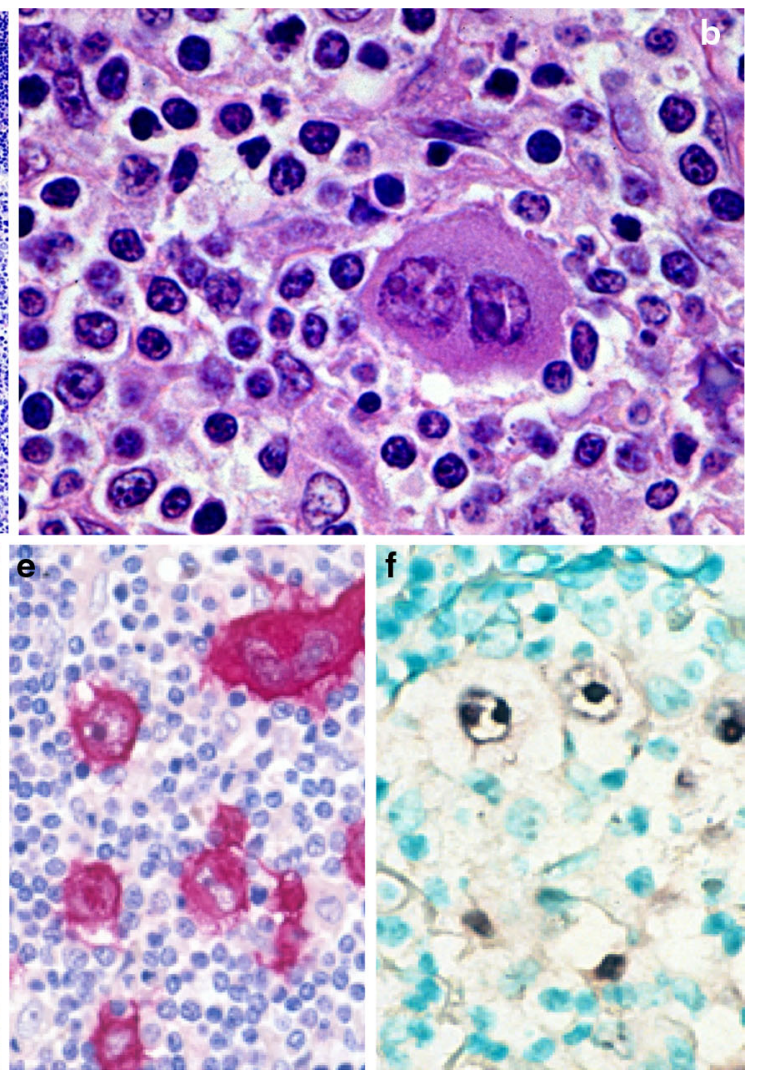

Hodgkin-like ATLL show Reed-Sternberg-like giant cells. The giant cells reacted with CD30 (c), CD15 (d) and EBV-LMP (e). The giant cells represented EBV-EBER1 RNA (f) 
Single sorted cell

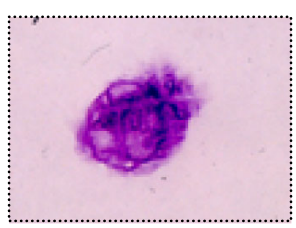

Giant cells

(CD30)

Single cell PCR: Ig JH

Giant cells (CD30)

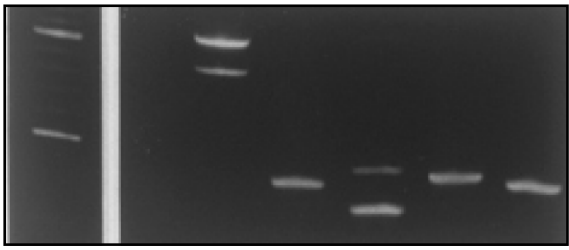

Fig. 3 A sorted single CD30-positive giant cell (HRS cell) (Giemsa staining). A sorted CD3-positive small cell (T lymphocyte) (Giemsa staining). The sorted CD30-positive giant cells morphologically closely resembled the HRS cells, while they were also single and not rosetted with lymphocytes. In addition, the sorted CD3-positive cells

Single cell PCR : HTLV-1 pX

$\begin{array}{ll}\text { T cell } & \text { CD3 } \\ & \text { CD4 } \\ & \text { CD8 } \\ \text { B cell } & \text { CD19 } \\ \text { Giant cells } & \text { CD30 }\end{array}$

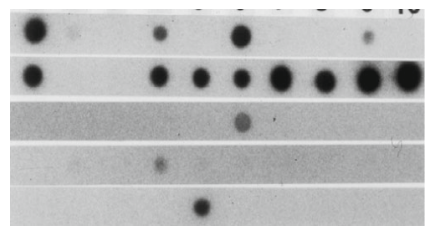

PCR (sorted100 cells) : TCR $\gamma$ JP

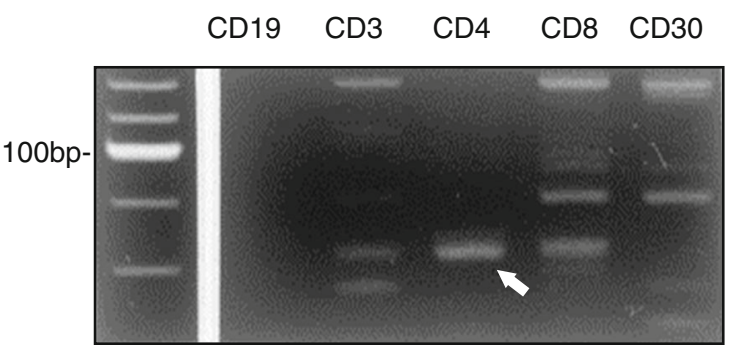

Fig. 4 In the single cell PCR analysis, HTLV-I of pX were amplified in the $\mathrm{T}(\mathrm{CD} 3$ and $\mathrm{CD} 4$-positive) cells, but were rarely observed in $\mathrm{B}$ (CD19), giant (CD30), and T (CD8) cells. The number of integrated HTLV-I cells was greater in the CD4-positive cells than in the CD3 cells. In an analysis of 100 sorted cells, B (CD19), T (CD3, CD4, CD8), or giant (CD30) cells were sorted and PCR was performed. The population of CD4-positive $\mathrm{T}$ cells showed monoclonal bands of $\mathrm{TCR}+$ (lane CD4). The CD3 (lane CD3), CD8 (lane CD8) or giant (lane CD30) cells represented polyclonal bands. The $\mathrm{B}$ cells (lane CD19) represented no bands

lymphocytes possess a peripheral $\mathrm{T}$ cell phenotype of helper/inducer cells $\left(\mathrm{CD}^{-}, 2^{+}, 3^{+}, 4^{+}, 8^{-}\right)$and that giant cells react with CD30 antibody and/or CD15. Receptor gene analysis has demonstrated the presence of rearrangement

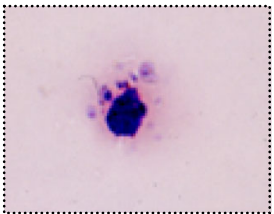

T cell

(CD3)

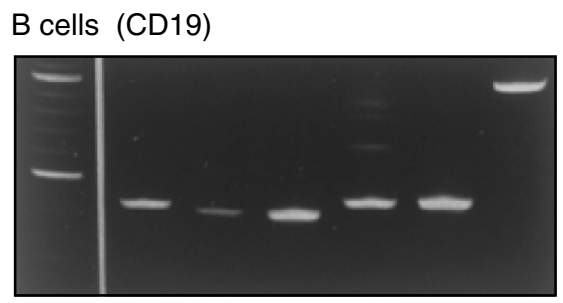

closely resembled the small lymphocytes. In the single cell PCR analysis, Ig JH was amplified in numerous giant (CD30) and B (CD19) cells. The giant cells showed one or two rearranged $\operatorname{IgH}$ genes. In addition, the PCR productions of Ig JH in the giant cells and $\mathrm{B}$ cells showed different lengths in each cell

and/or deletion of the TCR gene $\mathrm{C} \beta$ and/or $\mathrm{J} \gamma$. Proviral HTLV-1 DNA bands have been found, although these bands are weaker than those usually seen in typical ATLL, probably due to the small population of integrated HTLV-1 lymphocytes.

Molecular analysis of single cell PCR (polymerase chain reaction) has shown that the giant cells feature various products of $\mathrm{IgH}$, which closely resemble B cells. These findings support the notion that the giant cells are reactive cells of B cell origin (Fig. 3). In addition, while HTLV-1 was not detected in giant cells, HTLV-I was found to infect the $\mathrm{CD}^{+} \mathrm{T}$ cells, which demonstrated clonality (Fig. 4). Based on these findings, $\mathrm{CD} 4^{+}$cells can be considered to play an important role in ATLL tumorigenesis [10].

\section{Survival rates}

The median survival time (MST) and 2- and 5-year survival rates for the different groups of patients are shown in Fig. 3 . Patients with lymph nodes with Hodgkin's and pleomorphic small cell types show the highest MST and 2- and 5-year survival rates. The pleomorphic (medium and large cell) type, which displays features typical of ATLL, has been associated with a rapidly deteriorating survival curve during both the early and late stages of the disease (Fig. 5). The anaplastic large cell type and AILT type are also associated with a highly aggressive course since almost patients die within 2 years after diagnosis. Hodgkin's type was found to be associated with a progressive decrease in survival rate during an 8-year observation period. Pleomorphic small cell type lymphoma has been associated with an initial steep 
increase in mortality with the rate reaching a plateau during the mid- and late period of observation. On the other hand, all cases with lymphadenitis were still alive at the end of one study (Fig. 5) [11].

\section{FOXP3 and ATLL}

Adult T-cell leukemia/lymphoma cells are believed to be derived from post-thymic helper $\mathrm{T}$ cells with the phenotype of CD4(+) and CD25(+). However, ATLL cells are not

HTLV-I associated lymph node lesions

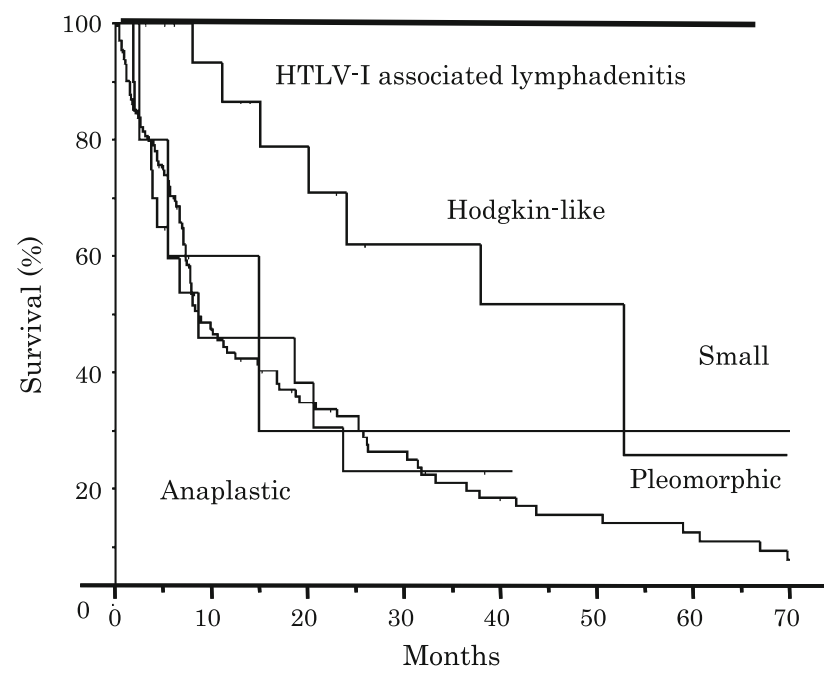

Fig. 5 Survival curve of HTLV-I associated lymph node lesions. The pleomorphic (medium and large cell) type $(n=219)$ and anaplastic large cell type $(n=27)$ are associated with a rapidly deteriorating survival curve, while Hodgkin's type $(n=18)$ shows a progressive decrease in survival rate. Pleomorphic small cell type $(n=18)$ is associated with an initial steep increase in mortality, reached a plateau during the middle and late periods of observation. In contrast, all cases with lymphadenitis $(n=14)$ were still alive at the end of the study concerned regarded as having a helper function; on the contrary, they have demonstrated a strong immunosuppressive activity in vitro [12]. In fact, ATLL is involved in opportunistic infections more frequently than other lymphoma, such as Pneumocystis carinii pneumonitis and strongyloidiasis [3].

Recent studies have characterized regulatory $\mathrm{T}$ cells, known as $T_{\text {regs }}$, in terms of coexpression of CD4 and CD25. Upon $\mathrm{T}$ cell receptor (TCR) stimulation, $T_{\text {regs }}$ potently suppress the activation/proliferation of other $\mathrm{CD}^{+} \mathrm{T}$ cells and $\mathrm{CD}^{+} \mathrm{T}$ cells in an antigen-nonspecific manner and in vivo usually serve to protect the host against the development of autoimmunity. FOXP3 represents a new T-cell marker considered to be more specific for $T_{\text {reg }}$ cells than other markers such as CD4 and CD25. Gene transfer of FOXP3 converts naïve CD4(+)CD25(-) T cells into a regulatory phenotype in both mice and humans, thus making this molecule a functionally important marker of the $T_{\text {reg }}$ cell population $[13,14]$.

We analyzed the expression of FOXP3, a key molecule of regulatory $\mathrm{T}$ cells, in nodal lesions of ATLL. Of 169 ATLL cases, 60 (36\%) showed FOXP3 expression in lymphoma cells (Fig. 6). Only one (5\%) of 22 cases with the anaplastic large cell variant expressed FOXP3, while $59 / 147(40 \%)$ of the pleomorphic cell variant cases did. Cytogenetic analysis showed that FOXP3(+) cases had simpler chromosomal abnormalities than FOXP3(-) cases, while clinically, FOXP3(+) cases and FOXP3(-) cases did not differ significantly in age distribution, clinical stage, lactate dehydrogenase and calcium in serum and overall survival. However, 8 of 34 FOXP3(+) cases showed a severe infectious state, which is an indication of immunosuppression, while only two cases of 62 FOXP3(-) cases did $(P<0.005)$. However, EB-virus-infected cells were significantly more frequently found in FOXP3(+) cases $(23 / 60 ; 38 \%)$ than in FOXP3(-) cases $(12 / 109$; $11 \%)(P<0.0001)$ (Fig. 6; Table 1). EBV-positive lymphoproliferative disorders (LPD) are often associated with
Fig. 6 FOXP3 expression in adult T-cell leukemia/lymphoma (ATLL). About $70 \%$ of lymphoma cells were positive for FOXP3 (a). But in some cases of ATLL, no FOXP3-positive cells were found (b). EB virus was detected by in situ hybridization. This figure shows scattered EBvirus-infected cells. EBER in situ hybridization (c)
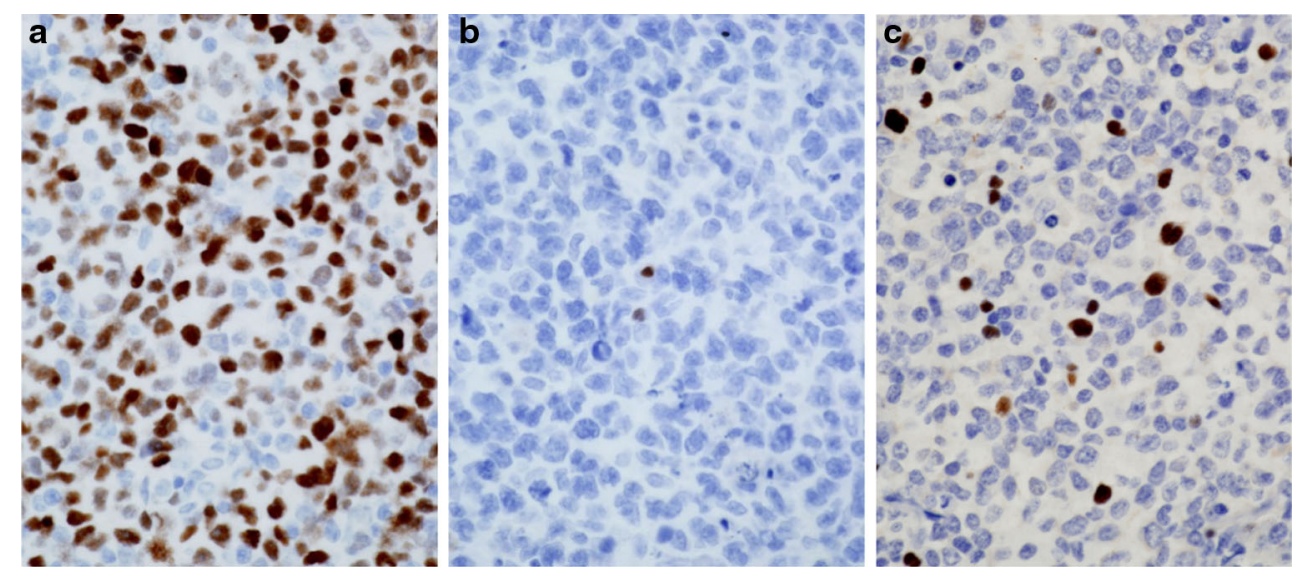
Table 1 Pathological features and FOXP3 expression in ATLL

\begin{tabular}{|c|c|c|c|c|}
\hline & & FOXP3(+) & FOXP3(-) & \\
\hline Morphology & $\operatorname{ALCL}(n=22)$ & 1 & 2177 & \multirow{3}{*}{$* *$} \\
\hline \multirow[t]{2}{*}{$(n=169)$} & Pleo, large $(n=76)$ & 23 & 53 & \\
\hline & Pleo, medium $(n=71)$ & 36 & $35] *$ & \\
\hline $\begin{array}{l}\text { EBER positivity } \\
(n=169)\end{array}$ & & $38 \%(23 / 60)$ & $11 \%(12 / 109)$ & $P<0.0001$ \\
\hline $\begin{array}{l}\text { CCR4 positivity } \\
(n=169)\end{array}$ & & $98 \%(59 / 60)$ & $93 \%(101 / 109)$ & $P=0.12$ \\
\hline $\begin{array}{l}\text { CCR8 positivity } \\
(n=86)\end{array}$ & & $32 \%(19 / 59)$ & $37 \%(10 / 27)$ & $P=0.66$ \\
\hline \multirow{4}{*}{$\begin{array}{l}\text { Other markers } \\
\text { positivity }(n=96)\end{array}$} & CD4 & $94 \%(34 / 36)$ & $98 \%(59 / 60)$ & $P=0.29$ \\
\hline & CD25 & $92 \%(33 / 36)$ & $83 \%(50 / 60)$ & $P=0.25$ \\
\hline & CD30 & $14 \%(5 / 36)$ & $27 \%(16 / 60)$ & $P=0.14$ \\
\hline & $\mathrm{CD} 4+\mathrm{CD} 25+$ & $83 \%(31 / 36)$ & $82 \%(49 / 60)$ & $P=0.57$ \\
\hline
\end{tabular}

${ }^{*} P<0.05,{ }^{*} * P<0.0005$

an immunodeficient state. In healthy carriers (Figs. 7, 8), EBV-positive cell proliferation is well regulated by EBVspecific cytotoxic $\mathrm{T}$ lymphocytes (CTL) [15]. In the immunodeficient state, however, EBV-infected cells proliferate accompanied by a decline in EBV-specific cytotoxic T-cell functions. In Hodgkin lymphoma, Hodgkin and Reed-Sternberg (HRS) cells are often infected with the EB virus, and regulatory T-cell infiltration around HRS cells coincides with the suppression of latent membrane antigenspecific $\mathrm{CD}^{+}{ }^{+} \mathrm{T}$-cell function and helps HRS cells to survive [16]. It is well known that EB-virus-infected cells, the majority of them $\mathrm{B}$ cells, emerge in peripheral $\mathrm{T}$ cell lymphoma types including ATLL [17]. The higher frequency of EBV-positive cells occurrence in FOXP3(+) ATLL than in FOXP3(-) cases indicates that FOXP3positive lymphoma cells may have a stronger suppressive effect on T-cell control of EBV-positive cells and thus induce their proliferation.

\section{CCL18/CX3CR1 and ATLL}

Chemokines are members of a family of small secreted proteins with some of them having received considerable attention because they display selectivity of cell targets and receptors, and are closely associated with T-cell subgroups. For example, the thymus and activation-regulated chemokine (TARC) is known to bind the CC chemokine receptor 4 (CCR4), which is expressed on activated Th2 lymphocytes. Yoshie et al. [18] reported a high frequency of CCR4 expression in ATLL and HTLV1 immortalized T cells. Moreover, CCR4 may facilitate ATLL-cell invasion of the lymph nodes. Hasegawa et al. [19] found that increased surface expression of CC chemokine receptor 7 (CCR7) correlated well with lymphoid organ involvement in ATLL.

To clarify the relationship between chemokines and their receptor expression, as well as the development of ATLL, 17 cases with ATLL were analyzed using DNA chips of chemokines and their receptors. All cases exhibited a varied and mixed pattern of up- and down-regulated gene expression of Th1, Th2, naïve and cytotoxic cellassociated chemokine genes. The clustering analysis of HTLV-1 associated lymphadenitis did not show any clustering pattern in either Hodgkin-like ATLL or ATLL. However, since CCL18 accounted for most up-regulated genes and CX3CR1 for most down-regulated genes, they were selected for immunohistochemical analysis. Immunohistochemical staining showed expression of the two genes of CX3CR1 and CCL18 in immunological cells, with a positive expression for reticulum cells and lymphocytes, but not for ATLL cells. HTLV-1 associated lymphadenitis type and Hodgkin-like type cases showed significantly higher CCL18 expression than did non-specific lymphadenitis cases. On the other hand, all HTLV-1 associated cases showed significantly lower CX3CR1 expression than did non-specific lymphadenitis cases [20].

Fractalkine/CX3CL1 is a membrane-bound chemokine that functions not only as a chemoattractant, but also as an adhesion molecule, and is expressed on endothelial cells activated by proinflammatory cytokines. The fractalkine receptor CX3CR1 is expressed on cytotoxic effector lymphocytes including NK cells and cytotoxic effector T cells, as well as on mature monocytes/macrophages and mucosal dendritic cells, all of which play important roles in the elimination of pathogens and cancer cells [21]. CCL18 is mainly expressed by a broad range of monocytes/macrophages and dendritic cells. Within a severe inflammatory environment, the CCL18-mediated attraction of naïve T cells toward the fully matured, interdigitating dendritic cells could assist in the mounting of a primary immune response. Enhanced CCL18 production has been demonstrated in several diseases, including various malignancies [22]. Immunohistochemical and in situ hybridization analysis showed that CCL18 was expressed by a subpopulation of tumor-associated macrophages (TAMs) that were preferentially located at the tumor invasion front, but not by neoplastic or non-neoplastic gastric mucosal cells [22].

In our study, the panel expression of chemokines and their receptors demonstrated that gene expression of CCL18 is up-regulated and CX3CR1 down-regulated compared with the gene expression in non-specific lymphadenitis [22]. Immunohistochemical staining shows that their expression occurs in immunological, but not in ATLL cells. These results suggest that an increase in CCL18 expression and a decrease in CX3CR1 expression are involved in immune responses against ATLL cells. 

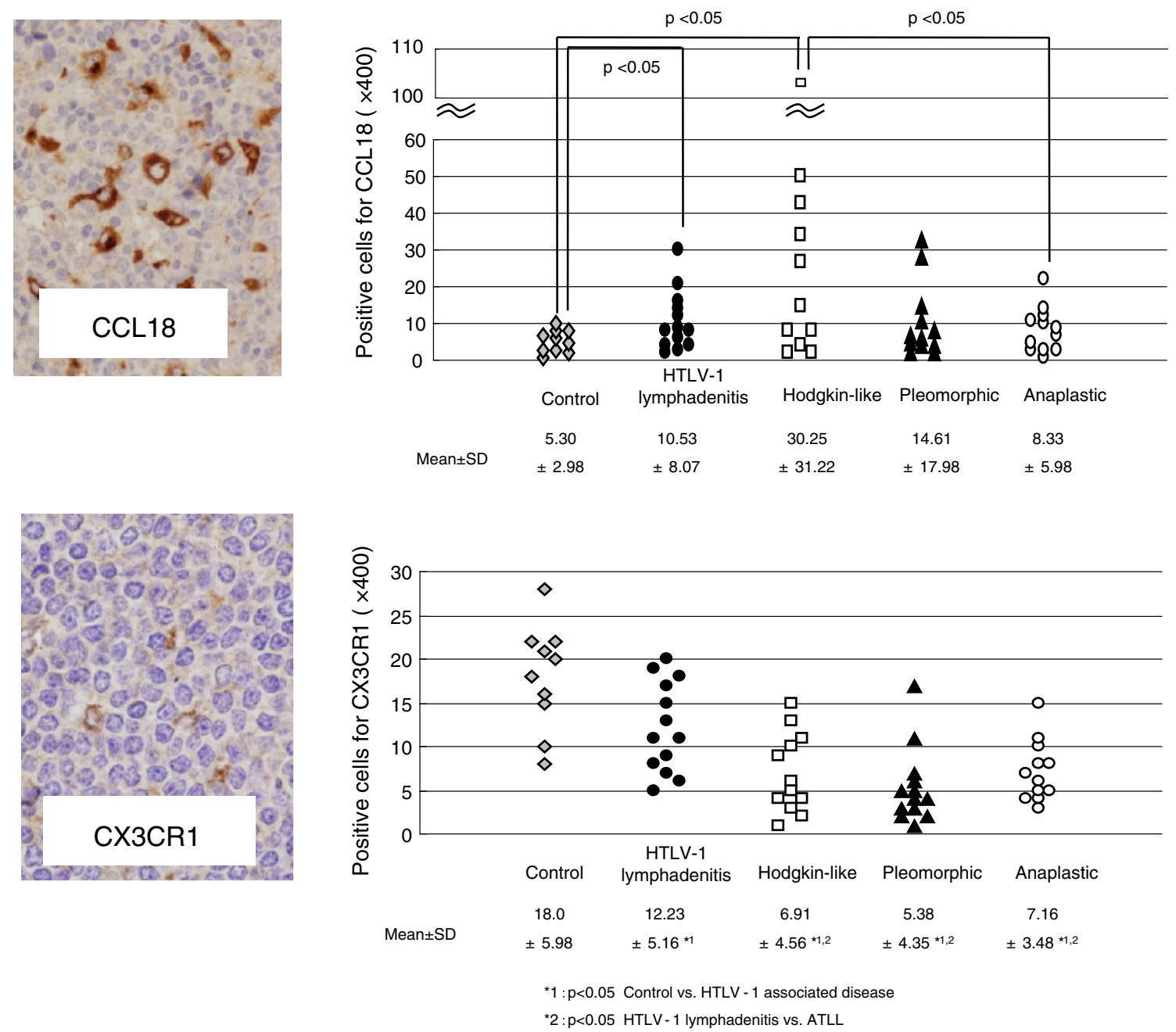

Fig. 7 Immunohistochemical staining for CCL18. The dendritic cells but not tumor cells were positive, while Hodgkin-like giant cells were also positive. Comparison of CCL18 expression in control cases (nonspecific lymphadenitis) and HTLV-1-associated diseases. HTLV-1associated lymphadenitis and Hodgkin-like type showed significantly higher expression than control cases (control versus HTLV-1associated lymphadenitis, $P=0.0046$; control versus Hodgkin-like

\section{TAMs and ATLL}

Tumor-associated macrophages (TAMs), which are known to possess the immunosuppressive M2 macrophage phenotype, contribute to tumor growth, invasion, and metastasis by producing various mediators. Macrophage polarization is divided into types, M1 and M2, based on the expression of membrane receptors, cytokines, and chemokines. M1 expresses CD80, interleukin (IL)-6, IL-12, and chemokine receptor 7, while M2 expresses CD163, IL10, and the chemokine ligand [23]. The classically activated M1 macrophages exhibit antitumor functions and the alternatively activated M2 macrophages protumor functions, which contribute to the development and progression type, $P=0.0018$ ). Immunohistochemical staining for CX3CR1. In pleomorphic type, the lymphoid cells were positive for CX3CR1 and were fewer than those in non-specific lymphadenitis. Comparison of CX3CR1 expression in control cases (non-specific lymphadenitis) and HTLV-1-associated diseases. All types of HTLV-1 associated diseases showed significantly lower expression than control cases

of tumors. CD163 expression has been associated with an anti-inflammatory M2 macrophage phenotype and is believed to be effective for distinguishing M2 macrophages from proinflammatory M1 macrophages [23].

For the in vitro study of ATLL, co-culture experiments using ATLL cell lines (ATN-1 and TL-Mor) and monocyte-derived macrophages were performed and BrdU incorporation of ATLL cell lines were found to be significantly elevated by direct co-culture with M2 macrophages. Analysis using the cytokine array indicated the presence of macrophage-derived soluble factors such as $\mathrm{C} 5 \mathrm{a}$, tumor necrosis factor (TNF)- $\alpha$, growth-related oncogene (GRO)$\alpha$, CCL1/I-309, and interleukin (IL)-6 stimulated ATLL cell lines. CD163 expression in macrophages was strongly 


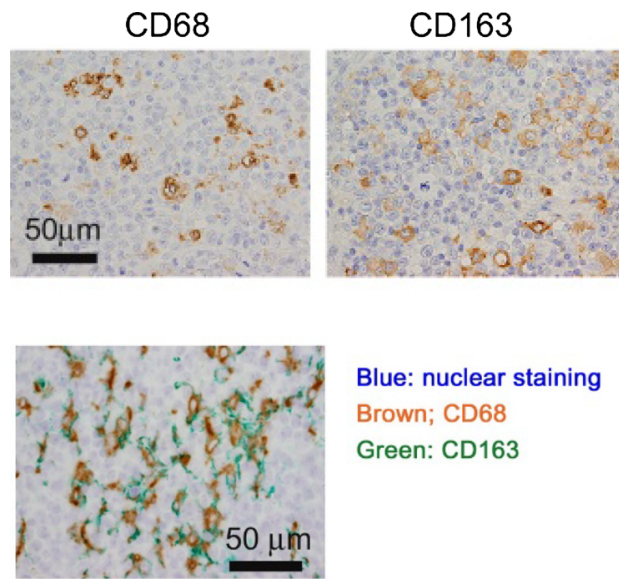

Fig. 8 Immunostaining of CD68 and CD163 in ATLL samples. Immunostaining of CD68 and CD163 were performed and the results of two patients were shown. Double-immunostaining of CD68 (brown colored) and CD163 (green colored) was done. Kaplan-Meier

induced by direct contact with ATN-1 cells, while downregulation of CD163 in macrophages significantly suppressed the growth of co-cultured ATN-1 cells. In addition, the number of CD68-positive macrophages in ATLL tissues did not correlate with overall survival $(P=0.25)$, whereas patients with a large number of CD163-positive macrophages ( $\geq 250$ cells $/ \mathrm{mm}^{2}$ tumor area; $n=37$ ) had worse outcomes than those with a small number $(<250$ cells $/ \mathrm{mm}^{2}$ tumor area; $\left.n=36\right)(P=0.05)$. Moreover, a higher ratio of CD163-positive to CD68-positive macrophages in ATLL significantly correlated with worse overall survival $(P=0.039)$, while multivariate analysis demonstrated that the number of CD163-positive macrophages constitutes an independent prognostic factor [24].

\section{Summary and conclusions}

Adult T-cell leukemia/lymphoma was first discovered in Japan, and isolation of the HTLV-1 retrovirus opened up a new, broad field of research in human lymphomagenesis. It also influenced histologic diagnosis, because a spectrum of morphologically diverse lymphoma has now been unified by the presence of the HTLV-1 proviral genome. By delineating the main microenvironmental dynamics that take place in ATLL, we aimed to convey the concept of the leading role performed by nonneoplastic lymphoma-associated immunological and stromal elements in influencing the natural history of ATLL, which so far has been considered to be little affected by the environment. The reciprocal effect of ATLL and its microenvironment results in the enhancement of the proliferative and invasive capabilities of the neoplastic clone and in the concurrent reshaping of the infiltrated tissues. The ATLL narrative is a

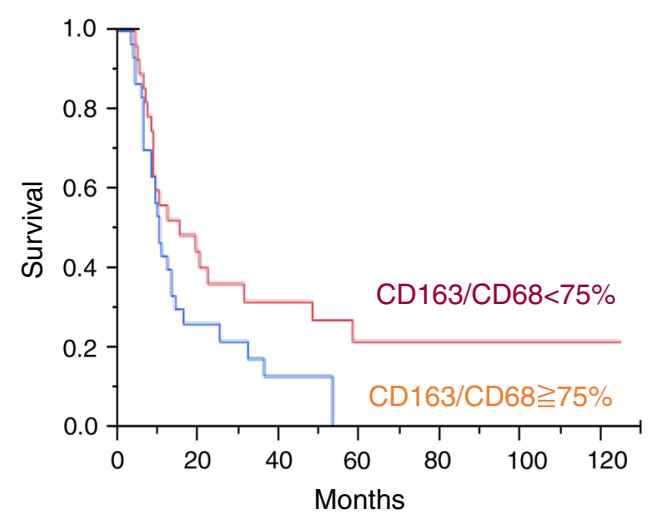

survival analysis of patients with high or low percentage of $\mathrm{CD}_{163^{+}}$cells in $\mathrm{CD}^{+} 8^{+}$cells was shown. The patients with high percentage of $\mathrm{CD}_{163}{ }^{+} \mathrm{M} 2$ macrophages showed poorer prognosis than low percentage

good example of an effective multidisciplinary collaborative approach by clinicians, pathologists, epidemiologists, and molecular biologists.

\section{References}

1. Takatsuki K. Overview of adult T-cell leukemia/lymphoma. Jpn Soc Res. 1985;25:97-103.

2. Yoshida M, Seiki M, Yamaguchi K, Takatsuki K. Monoclonal integration of human $\mathrm{T}$-cell leukemia provirus in all primary tumors of adult T-cell leukemia suggests causative role of human T-cell leukemia virus in the disease. Proc Natl Acad Sci USA. 1984;81:2534-7.

3. Takatsuki K, Yamaguchi K, Watanabe T. Adult T-cell leukemia and HTLV-I related diseases. Gann Mono Can Res. 1992; 39:1-15.

4. Ohshima K, Kikuchi M, Yoshida T, Masuda Y, Kimura N. Lymph nodes in incipient adult T-cell leukemia lymphoma with Hodgkin's disease-like histologic features. Cancer. 1991;67: 1622-8.

5. Ohshima K, Kikuchi M, Masuda Y. HTLV-I associated lymphadenitis. Cancer. 1992;69:239-48.

6. de Jong D, Enblad G. Inflammatory cells and immune microenvironment in malignant lymphoma. J Intern Med. 2008;264: 528-36.

7. Cacciatore M, Guarnotta C, Calvaruso M, Sangaletti S, Florena $\mathrm{AM}$, et al. Microenvironment-centred dynamics in aggressive B-cell lymphomas. Adv Hematol. 2012;2012:138079. doi:10. 1155/2012/138079.

8. Ohshima K, Suzumiya J, Sato K, Kanda M, Sugihara M, et al. Nodal T-cell lymphoma in an HTLV-I endemic area; proviral HTLV-I DNA, histological classification and clinical evaluation. Br J Haematol. 1998;101:703-11.

9. Karube K, Suzumiya J, Okamoto M, Takeshita M, Maeda K, et al. Adult $\mathrm{T}$ cell lymphoma/leukemia with angioimmunoblastic $\mathrm{T}$ cell lymphoma-like features: report of 11 cases. Am J Surg Pathol. 2007;31:216-23.

10. Ohshima K, Suzumiya J, Kato A, Tashiro K, Kikuchi M. Clonal HTLV-I-infected CD4+ lymphocytes and non-clonal non- 
HTLV-I-infected giant cells in incipient ATLL with Hodgkin-like histologic features. Int J Cancer. 1997;72:592-8.

11. Ohshima K, Suzumiya J, Sato M, Kanda M, Simazaki T, et al. Survival of patients with HTLV-I-associated lymph node lesions. J Pathol. 1999;189:539-45.

12. Yamada Y. Phenotypic and functional analysis of leukemic cells from 16 patients with adult T-cell leukemia/lymphoma. Blood. 1983;61:192-9.

13. Khattri R, Cox T, Yasayko SA, Ramsdell F. An essential role for Scurfin in CD4+ CD25+ T regulatory cells. Nat Immunol. 2003;4:337-42.

14. Hori S, Sakaguchi S. Foxp3: a critical regulator of the development and function of regulatory T cells. Microbes Infect. 2004; 6:745-51.

15. Karube K, Ohshima K, Tsuchiya T, Yamaguchi T, Kawano R, et al. Expression of FoxP3, a key molecule in CD4+ CD25+ regulatory $\mathrm{T}$ cells, in adult $\mathrm{T}$-cell leukemia/lymphoma cells. $\mathrm{Br} \mathrm{J}$ Haematol. 2004;126:81-4.

16. Ishida $\mathrm{T}$, Ishii $\mathrm{T}$, Inagaki $\mathrm{A}$, Yano $\mathrm{H}$, Komatsu $\mathrm{H}$, et al. Specific recruitment of $\mathrm{CC}$ chemokine receptor 4-positive regulatory $\mathrm{T}$ cells in Hodgkin lymphoma fosters immune privilege. Cancer Res. 2006;66:5716-22.

17. Ho JW, Ho FC, Chan AC, Liang RH, Srivastava G. Frequent detection of Epstein-Barr virus-infected B cells in peripheral T-cell lymphomas. J Pathol. 1998;185:79-85.

18. Yoshie O, Fujisawa R, Nakayama T, Harasawa H, Tago H, et al. Frequent expression of CCR4 in adult T-cell leukemia and human T-cell leukemia virus type 1-transformed T cells. Blood. 2002; 99:1505-11.
19. Hasegawa H, Nomura T, Kohno M, Tateishi N, Suzuki Y, et al. Increased chemokine receptor CCR7/EBI1 expression enhances the infiltration of lymphoid organs by adult T-cell leukemia cells. Blood. 2000;95:30-8.

20. Shimizu K, Karube K, Arakawa F, Nomura Y, Komatani H, et al. Upregulation of $\mathrm{CC}$ chemokine ligand 18 and downregulation of CX3C chemokine receptor 1 expression in human T-cell leukemia virus type 1-associated lymph node lesions: results of chemokine and chemokine receptor DNA chip analysis. Cancer Sci. 2007;98:1875-80.

21. Nishimura M, Umehara H, Nakayama T, Yoneda O, Hieshima K, et al. Dual functions of fractalkine/CX3C ligand 1 in trafficking of perforin+/granzyme B+ cytotoxic effector lymphocytes that are defined by CX3CR1 expression. J Immunol. 2002;168: 6173-80.

22. Leung SY, Yuen ST, Chu KM, Li R, Chan AS, et al. Expression profiling identifies chemokine (C-C motif) ligand 18 as an independent prognostic indicator in gastric cancer. Gastroenterology. 2004;127:457-69.

23. Komohara Y, Horlad H, Ohnishi K, Ohta K, Makino K, et al. M2 macrophage/microglial cells induce activation of Stat 3 in primary central nervous system lymphoma. J Clin Exp Hematop. 2011; 51:93-9.

24. Komohara Y, Niino D, Saito Y, Ohnishi K, Horlad H, et al. Clinical significance of CD163+ tumor-associated macrophages in patients with adult T-cell leukemia/lymphoma. Cancer Sci. 2013;104:945-51. 